Article

\title{
Empirical Study of Communication of Audience Cognition of Environmental Awareness
}

\author{
Jun Wu ${ }^{1,2, *}$, Yang Gao ${ }^{2}$, Sang-Bing Tsai ${ }^{3,4,5}$ (D) and Rungtai Lin ${ }^{2}$ \\ 1 Department of Animation, School of Journalism \& Communication, Anhui Normal University, \\ Wuhu 241002, China \\ 2 Affiliation Graduate School of Creative Industry Design, National Taiwan University of Arts, \\ Xinpei 22058, Taiwan; lukegao1991@gmail.com(Y.G.); rtlin@mail.ntua.edu.tw (R.L.) \\ 3 Zhongshan Institute, University of Electronic Science and Technology of China, Zhongshan 528400, China; \\ sangbing@hotmail.com \\ 4 College of Business Administration, Capital University of Economics and Business, Beijing 100070, China \\ 5 Research Center for Environment and Sustainable Development of China Civil Aviation, \\ Civil Aviation University of China, Tianjin 300300, China \\ * Correspondence: junwu2006@hotmail.com
}

Received: 26 March 2018; Accepted: 25 May 2018; Published: 30 May 2018

check for updates

\begin{abstract}
Environmental protection is a vital issue nowadays. Many scholars typically focused on the social aspects of it in their research. For the practical implementation of environmental protection, raising environmental awareness is crucial. With the rapid development of digital media, animation is now used to effectively convey this issue of concern. This study employed qualitative and quantitative methods to explore the effects of viewers' esthetic experiences and preferences for an original environmental protection-themed sand animation in order to understand cognitive differences between viewers regarding film and television. We selected critical social issues and a popular sand animation for this study. Analyzing the cognitive differences and preferences of the viewers, we identified key factors that attract viewers. The findings were as follows: (1) People gain clear cognition of the environmental protection theme from the sand animation. (2) Innovative perspectives gain recognition more easily. (3) Propagation of environmental awareness to audiences is possible through fiction. (4) Age and professional background cause differences in cognition of film by audience. (5) Different elements of film have different effects on creative intensity and cultural depth.
\end{abstract}

Keywords: environmental protection; audience awareness; animation; environmental issues; sustainability

\section{Introduction}

Issues, contradictions, and conflicts elicited by the deteriorating global environment in the industrial age are increasing, along with the social problems they cause. Liu noted that, "the environmental issue has become a global public issue and has an in-depth impact on the structure and order in other areas of society" [1]. The global environment has become a theme. Countries and organizations such as the United States, Japan, South Korea, India, Brazil, and the European Union have proposed green development strategies. As noted previously, "The increase in the number of people far exceeds the extent that the natural ecosystems can support. The basic necessities of life, as well as the attendant and ever-increasing demands for entertainment and luxury, are all fundamentally disturbing and undermining the natural ecology system, resulting in an irreversible reduction in the quantity and quality of resources and energy" [2].

Environmental animation, an animated work that reflects the thoughts of humans and the concept of environmental protection [3], in the context of the global ecological crisis has a high 
public welfare value. Environmental animation is a manifestation of environmental transmission, a universal expression of environmental awareness. Environmentalism and ecologism have different meanings. In ecological animation, biomorphism and ecocentrism construct multiple ecospheres. Anthropocentrism, biocentrism, and ecocentrism present different environmental ideologies and esthetic characteristics through the construction of visual imagery in environmental film [4]. The audience can reflect on the discourse logic and fully fledged relationships carried by form in the experience of image narration [4]. The animation employs imagery as a carrier to integrate the key topic into the narration and express current environmental concerns in a critical manner through audiovisual arts, thereby reminding people to reexamine the relationship between humans and nature. Some discuss the dismal truth in documentaries. Some predict future disaster. Some arouse thinking through the use of irony [5]. The crucial contribution of environmental films lies in the spread of environmental awareness and ecological ideas in a manner favored by the audience to communicate effectively and avoid inner contradiction between traditional education about environmental protection and technical barriers [4]. The ecological ideas disseminated through environmental films are crucial to establishing and passing on the intended environmental ethics to the public [5]. As a mass medium, animation has a unique esthetic value and social function. The aesthetic value: The aesthetic tension between strange animation images and people's habitual thinking; the aesthetic tension of the aesthetic form created by animation; fairy-tale aesthetic sentiment [6]. The social functions are those of enlightenment, power, cognition, information, esthetics, and entertainment [7]. It makes use of the psychology of human cognition, curiosity, subordination, and empathy to achieve social education through the selective processing of the audience's consciousness to understand and adapt to the world [5].

Existing research on environmental issues emphasizes the morphology of the works, and the narrator is rarely seen from the audience's perspective. Therefore, it is necessary to further explore the specific role that environmental awareness plays among film audiences. This study investigated an audience's concepts of environmental protection after watching an animated film and solicited preferences for the form of transmission through a qualitative interview method [8]. Questionnaires were used to survey the general recognition of the film in order to provide a reference to agencies and workers for the dissemination of environmental issues. This study explored the relationship between artists and audiences in intercultural communication. This process can be used to analyze and explain other communication media by constructing and validating cognitive factors that affect artwork. This research project is one of a series on film and television animation, using the original sand animation, also known as sand art, a form of visual storytelling in which an artist dexterously manipulates fine granules of sand to produce images and animations. The process begins by applying sand to a lighted surface, after which images are rendered on the surface by drawing lines and figures with bare hands. $[9,10]$. The sand animations discussed in this article refer to the use of sand (or fine particulate matters such as flour and salt) and framing techniques to capture a series of images and create an animation, but excludes sand paintings and sand performance videos. [11,12]. With the aid of the animation "Statue of Blood", this research explored audience cognition of environmental issues, which mainly included the following aspects:

1. Depth of recognition of the environmental theme of different viewers after watching the animation.

2. Audience cognition of different forms and perspectives of video animation.

3. Cognitive differences in the evaluation of film elements among audiences from different backgrounds.

4. The influences of elements of film and television works on the audience's overall cognitive assessment. 


\section{Research Framework}

\subsection{Literature Review}

In 2004, film scholar Scott MacDonald introduced the concept of "eco-cinema" in his book Constructing Ecological Movies. Eco-cinema refers to the viewing path of contemporary society in which the subject is increasingly infiltrated and shaped by the commercial logic of the image [5]. The concept of eco-cinema is derived from eco-literary criticism. According to American scholar Lawrence Buell, the term "eco-criticism" first appeared in an essay published by American literary critic William Rucker in 1978. It was not until the early 1990s that eco-criticism was accepted by American and British scholars [13]. Environmental image refers to the integration of ecological awareness into the plot of a film, in which the image serves a setting opposing consumer culture and cultural struggles. An environmental image contains either praises, criticisms, or warning messages; through the film lens, it draws people's attention and prompt them to reflect upon the problems of the ecosystem [14,15].

Audience is the key to understanding art, because the meaning that art creates depends on how it is used by its consumers, rather than by creators [16]. Art shapes the world through its audience, and most viewers reach the appropriate understanding [17]; in other words, members of the audience are able to interpret works of art based on their personal experiences. Their interpretations differ from the original intention of an artist's work. Everyone has different interpretations of the meaning of cultural texts, they have strong initiative in the creation of meaning [18]. Hall stated that art objects are regarded as "texts" of meaning that are displayed in codes of language and vision. The creators of art objects compile meaning into them, and people can utilize different methods of decoding to interpret unique meanings from this text [19]. Edmund Burke revealed that if what appears in works of art inspires the audience to explore it in reality, the core strength of the works lies in the subject of the art itself, rather than in imitative or realistic techniques [20].

McLuhan indicated that any influence on individuals and society through any medium (i.e., any extension of person) is caused by a new dimension; any extension of ours (or any new technique) introduces a new dimension. The content of any medium is another type of medium. "The medium is the message." The medium shapes and controls the scale and form of human combination and action. The message of film media is a transition from a linear connection to a contouring [21]. Lasswell proposed the five Ws of dissemination: Who says what to whom through which channel to what effect? Effect analysis is the goal of mass communication research. The methods for achieving this effect are also a focus of mass communication [22].

\subsection{Photo-Elicitation Interviews}

Harper [23], Wang and Burris [24], and other scholars pointed out that photo-elicitation interviews can use images to help interviewees to recall and reflect on their personal experiences and impressions, helping themselves to understand the subjective awareness.

Collier and Collier [25] pointed out that images and sounds can carry more information, including human emotions. The researchers record the data by observation, emphasizing the importance of observation and images.

\subsection{Literature Review}

Art creation is a process of continuous exchange. When evaluating works, we must understand the communication between artists and audiences, not only in the social context, but also in the interactive experience between artists and audiences [26,27]. Lin et al. [28] constructed a theoretical framework for product designers to communicate with users during the design process. For the cognitive evaluation of artwork, a corresponding research framework has been introduced to describe the emotional response of poems conveyed through paintings and the cognition of those visual scenes [29,30]. In addition, these research frameworks have proved effective in converting poems to paintings [31]. Lin et al. [32] combined communication theory and mental models along with the 
cognitive theory of communication studies to construct a research framework for evaluating film and television, as shown in Figure 1.

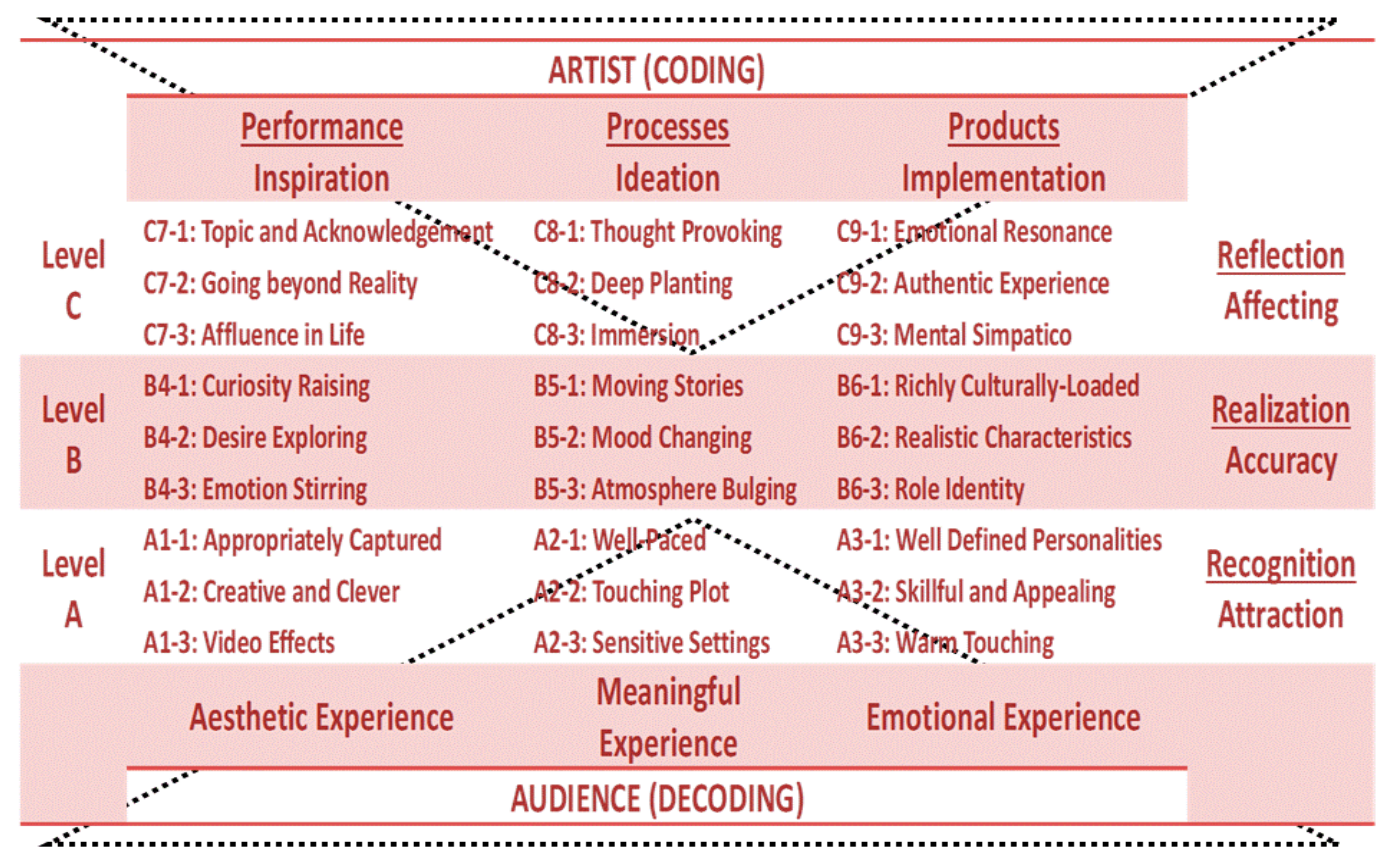

Figure 1. Attributes for evaluating artwork [22].

Artists create works by combining different external elements (e.g., lens, special effects, and sounds) together, thereby forming a complete film. This is an encoding process. Viewing by the audiences is a deciphering and decoding process, wherein viewers watch, understand, and become moved by the film.

\subsection{Research Framework for Evaluating Microfilm}

Based on previous studies [28,32-35], a cross-cultural design research architecture was constructed by combining communication theory and mental models (Figure 2). Cross-cultural research is based on science, art, and design. Design communication must be expressed in three key stages by the designer: performance (inspiration), process (ideation), and product (implementation). Performance is the source of inspiration; the designer's ideas can be conveyed through the design of the performance. The design process is how the designer's idea is reproduced through imagination, thoughts, and emotions. Products are the realization of inner thoughts and expressions. When the designer's thoughts are consistent with the consumer's, they can be more easily conveyed [28,36-38].

From a communication standpoint, the design of a product based on symbology must fulfill three functions: The functions of communication must be satisfied, and an effective communication must encompass both external expression and internal meaning. In the CHEER model, its properties were explored and identified as a framework for evaluating product design [32]. Norman [39] described three levels: designing intrinsic, behavioral, and design reflections and representing the esthetics, meaning, and emotion of the user experience [28,37]. These experiences can be used to evaluate product design and determine the three goals of product design: recognition (attracting), realization (accuracy), and reflection (affecting), as shown in Figure 2 [29,30]. 


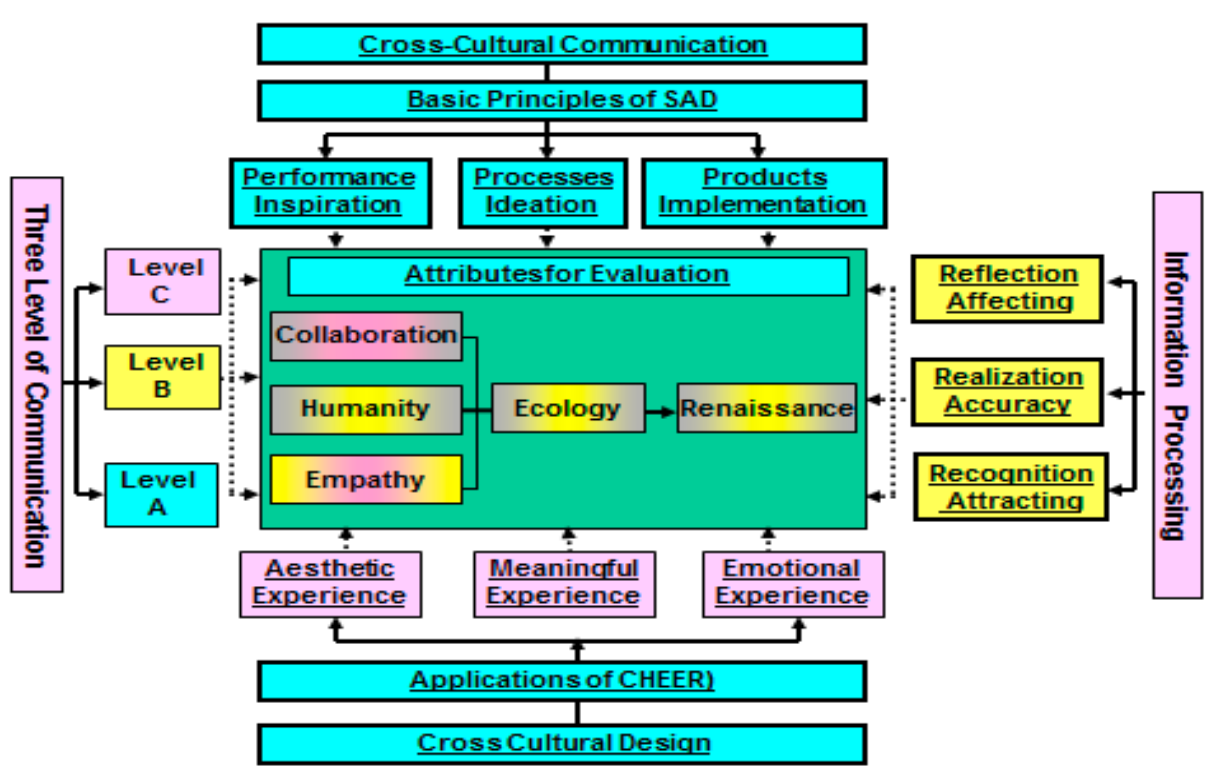

Figure 2. Cross-cultural communication model (Lin et al., 2018).

\section{Research Design and Implementation}

\subsection{Research Methods}

This study used empirical research, questionnaires, and in-depth interviews to obtain information. Through questionnaires issued to a random sample, a diverse audience's cognition of a sand animation film was explored. The researchers observed and recorded the participants' perception of the film unobtrusively. The 10 participants in the in-depth interviews were numbered A1 to A10. The interview transcripts were analyzed by investigators one by one to identify and encode relevant concepts; they carefully examined the structures within the concepts and integrated concepts with similar characteristics into categories, followed by examining the relationship among categories to form research-related topics. Quantitative data analysis involved the independent samples' $t$ test, analysis of variance, multiple regression, and other methods. Regarding qualitative research, a relationship was formed between argument and echoing to inspect the differences in the results of in-depth interviews and form general conclusions.

\subsection{Questionnaire Design}

The questionnaire comprised questions about basic information and animation assessments to determine whether the intended environmental theme in the sand animation was recognized by the audience. Lin et al. [32] constructed a cognition structure of movie and television animation. Film language, plot, sound, and image features are used by the creator to encode the message, and the viewers decode the message and generate emotions after viewing with the help of external elements of the work. Basic information consisted of gender, age, and professional background. There were four parts of the animation assessment: (1) Did the audience see it? Nine questions surveyed opinions on the use of VFX, use of film language, sound creation techniques, design of the plot, construction of a spatial environment, use of rhythm, image feature presentation, presentation skills, and skills of emotional expression. (2) Did the audience understand the message? Nine questions determined thoughts on how to stimulate the imagination, how to integrate the pieces, the degree of emotional appeal, the development of the story, the creation of the atmosphere, and the display of the mood changes. (3) Was the audience touched by it? Nine questions identified feelings about the presentation of personality traits, display of life experience, interpretation of role identity, symbol of artistic conception, feeling of true experience, transcendence of reality, theme communication, 
immersive relationship, empathetic interaction, and deep-rooted sympathy. (4) Finally, three questions solicited general comments on the depth of culture, level of creativity, and preferences of works. The five-point scale ( 5 being the highest) was explained to the on-site participants before the questions were answered. The questionnaire was completed through a mobile phone network and accessed by $\mathrm{QR}$ code. Assistance was provided by on-site staff in case of difficulties in mobile phone operation. The website is https://goo.gl/forms/tFsBtGQHs7zsBRBu2.

For the in-depth interviews, 10 participants were invited. The 10 samples were all invited from participants who participated in the empirical research, with different professional backgrounds and occupations as segments. These 10 invited persons all have rich experience in relevant fields. Their mutual confirmation of their responses to the theories of the research framework constitute the basis of this research. The interviews were conducted one-on-one. The researchers conducted the open-structured interviews and recorded them with the consent of the participants, and a verbatim manuscript was produced. The results were submitted to the interviewees for confirmation. If there was any doubt, additional amendments were made. Finally, the keywords were extracted and classified. The following 10 questions were asked:

Q1: In your opinion, what is the theme of the film?

Q2: After watching the film, what do you think about this topic?

Q3: In your opinion, what should we do about this topic in our daily life?

Q4: What impressed you in the film?

Q5: What do you think about telling the story from an animal's perspective in the film? Why?

Q6: The whole film is connected by birds, have you noticed that? How do you think about it?

Q7: Do you think it is controversial or more conducive to the elaboration of the theme using Buddha statues as a reflection of animal protection?

Q8: Many fictional images are used in the video. What do you think is the difference from reality? What kind of expression do you prefer and why?

Q9: What do you think is the difference between using sand animation and using pictures and painting for expression?

Q10: Do you think that use of realistic media facilitates expressing the meaning of a topic?

\subsection{Research Participants}

The research participants in this study watched the sand animation "Statue of Blood". ("Statue of Blood" announces the connection of all beings and that life and death are a profound and undeniable theme in nature. Greedy people destroy this harmony in pursuit of their own selfish desires. They try to carve their own beliefs onto the bloody ivory. They do not know or care that the production of ivory Buddhist statues and beads means the end of an elephant's life. This sand cartoon film shows the process of the production of ivory Buddhist statues and beads. It also uses metaphorical techniques to show the relationship between humans and nature. Hopefully, it will give viewers a desire for concern and vigilance in order to challenge the world to refuse to deal with the damage the trade creates, and to teach respect and love for other life forms and nature itself). For the in-depth interviews, 10 members of the audience were invited. Among them, three had a professional background in film and television animation, four had a background in art design, and three had other professional backgrounds. Occupations comprised three university teachers, four general workers, and three students. A total of 140 people completed the questionnaire after watching the video. Of these, 99 were women, 41 were men, 62 were aged younger than 19 years, 56 were aged between 20 and 29 years, nine were aged older than 50 years, seven were aged between 30 and 39 years, and five were aged between 40 and 49 years. The professional backgrounds comprised 63 design-related professionals, 35 art-related professionals, 24 film and television animation-related professionals, 11 other professionals, and six music-related professionals. 


\subsection{Research Implementation}

Participants were invited to the laboratory 30 at a time through random sampling. Through five sessions, 140 people participated. It was explained to participants that a film of $4 \mathrm{~min}$ and $50 \mathrm{~s}$ would be shown. After the movie, people were invited to complete the questionnaire. This study assumed that the dissemination of environmental awareness is more concentrated in the education system than in the general public. Therefore, through intensity sampling, three groups of teachers, students, and ordinary workers were selected. Through random sampling, questionnaire participants were invited for an interview after providing consent. A total of 10 participants completed in-depth interviews.

\section{Results and Discussion}

In this study, 10 viewers were invited to discuss the effect of sand animation on the dissemination of environmental themes and preferences for different perspectives and forms. We reviewed and formed connections and comparisons between the in-depth interview results and the cognition results of all 140 participants.

\subsection{Viewer Awareness of the Theme of Environmental Protection}

Of the 10 interviewees, five interpreted the theme of the film as the protection of animals, two considered the theme as both protecting the environment and caring for animals, and one considered it a religious theme. Of the 10 we interviewed, nine regarded it as environmentally related with higher theme awareness. People generally perceived the topic to be significant, important, and profound in meaning; and two considered it to be consciously directed at people to evoke more profound thinking by "arousing human beings' respect and awe for nature" (A2); "constantly reflecting on [themselves] and [showing that] the essence of environmental protection is human self-help and self-preservation" (A3); showing that "the way to make people's environmental awareness into environmental action is the most important" (A4); and alerting "people to get away from desire and have eternal life with nature" (A10). This indicated that the theme of environmental protection is likely to cause cognitive resonance.

Understanding the effect of the action on the theme through cognition of the theme is an essential element of evaluating the effectiveness of communication. The answers of the 10 interviewees about the theme were mainly focused on four dimensions: awareness, action, education, and philosophy. Awareness level: it is generally believed that humans should care for and protect the environment and should not arbitrarily kill animals or waste resources. Action level: we should use environmentally friendly materials when possible, participate in animal welfare activities, provide help to stray cats and dogs, and refuse to buy ivory. Educational level: "We should focus on children's environmental education. Animation is the best form to raise children's environmental awareness" (A4). "Through the film and other perceptual ways, we should influence more and more people to value environmental protection" (A6). A5 said, "Salvation of the sins of the killer by doctrine." Conceptual level: A3 mentioned, "Thinking of the environment as part of life." A7 emphasized, "Caring for life, regarding life equally always with awe and respect for life." The interviewees had not only a strong sense of environmental protection and relevant plans of action in their lives, but also thoughts on the educational level of environmental protection. They also had corresponding philosophical thoughts on the concepts of life.

\subsection{Viewer Cognition of the Film Perspective}

The implications of the cognition of the 10 participants interviewed on the sand animation film were explored. The film impressed most with its direct picture reproduction: as the elephant opens his eyes and sees someone sawing his teeth (A1), the Buddha's head falls (A7), the lawbreakers shed tears (A3, A5), and elephants treat human killings with forgiveness rather than vengeance (A2, A4, A6, A8). The participants were impressed by the tragedy of the elephants and their forgiveness of the humans. 
In particular, people were impressed that the elephants still do not wish to hurt people despite some being killed by hunters. There were also deep thoughts on meaning, such as "a series of thinking of buying and selling, death, selfishness, life, Buddhism, equality, and nature extending from the death of 'Elephant' and the birth of 'Like'" (A10).

Animals are the most prominent theme in animated films [40]. Animals can stimulate the most primitive emotions and imagination within human beings. However, people are self-centered and tend to cast themselves onto nonhuman objects. Therefore, most animals in animation are personified to express the subjective thinking of humans. The film in this study abandons the human perspective and explores the perspective of animals to understand their situation. This study explored whether this shift of focus was accepted by the audiences. All the participants considered it to be highly appropriate and shocking. A3 stated, "Very good, the perspective abandons the long-held, people-centered view, leaving 'it' to be the center and human to be supporting actor, which makes it more realistic". "It's good and makes the viewer be more sympathetic with the situation of the animals and also exerts an animated presentation advantage" (A4). A6 said of the shift of focus, "Very good, such an expression touches the mind more intuitively." The film producer deliberately connects the plot with birds, which appear three times. Of the 10 interviewed participants, five failed to notice this, and their understanding was also different, assuming the themes to include nature, freedom, divinity, and God.

Buddhism aims to promote respect for life and God with the purpose of delivering all living creatures from torment and performing good deeds. The sand animation in the study utilizes the metaphor of Buddhism to convey the issue of environmental protection and uses the production of ivory beads and statues as a connection. The study explored whether these were acknowledged by the participants. Of the 10 participants, five considered it to be appropriate and to not cause controversy reflecting animal protection issues with the Buddha image. The other five thought that there would be some controversy, but that it would be more conducive to the elaboration of the theme. "It will be controversial but more conducive to the elaboration of the theme, the binding force of religion can more effectively change people's thinking and behavior" (A4).

\subsection{Cognition on the Film Form}

When people refer to environmental issues, people will think of the phenomenon or behavior that destroys the environment in real life. An authentic image rich in details can carry greater amount of information, which easily prompts people to establish associations with reality more intuitively through their visual and hearing senses. However, the representation of these issues through sand animation uses fictional images. Discussing whether a fictional image can convey true meaning and cause emotional resonance is crucial. Of the 10 participants we interviewed, two liked both the fictional and real nature, and one noted that, "fiction is more mysterious and expressive, nature is more authentic, rustic, and life-savvy" (A4). The remaining eight reported preferring fiction, "[because of the] sublimation of specific things in life, stimulation of people with more emotion will obtain more emotional comfort, more impact" (A3); "fiction gives more imagination space" (A5, A8); "[fiction is] more abstract, more concise" (A7); and "[fiction is] avoiding the bloody shots of the real scene, enriching the movie while echoing the theme" (A10).

The differences among sand animation, painting, and photos were further explored. The participants provided accurate descriptions of the differences among the three media. Sand animation was perceived as more lively, dynamic, mysterious, and flexible as well as attentive to methods, compared with painting and photos (A3). "Sand animation and painting are not conducive to the presentation of details, but conducive to plot narrative, and the photo is more conducive to the theme expression" (A7). "Compared with painting and photos, the glowing sense is stronger and the expression is more subtle and esthetic. This kind of theme can be overwhelming and resentful if photos are used" (A8). These statements reveal that sand animation, painting, and photos have their own advantages for conveying environmental issues, but the audience preferred sand animation because it is artistic, creative, implicit, and esthetic. 
Realism tends to bring a true sense of experience to the audience and explore the relationship between the materiality of the medium and the expression of the theme. Of the 10 participants, four believed there was a relationship between them. They thought that the realistic presentation of the medium was tangible and brought people into the plot to further understand the topic. The other six people assumed that the realism of the medium had little relation to the expression of the theme; as long as the theme can be expressed thoroughly, the form of expression can vary.

\subsection{Cognitive Differences between Audience Members of Different Ages}

Validity analysis of 140 questionnaire results revealed a high KMO coefficient of 0.953 and a strong significance value of 0.000 . The eigenvalue was 9.159 , which can explain $64.946 \%$ of the variance for the default purpose. The load of each question ranged from -0.286 to 0.433 , and the commonality ranged from 0.23 to 0.785 . Testing the reliability of the questionnaire entailed examining the internal consistency of all dimensions of the scale and the reduction of Cronbach's $\alpha$ after the deletion of a single item. The questionnaire analysis revealed that Cronbach's $\alpha$ was 0.980 . The correlative corrections of the facets and the contents of single facets ranged from 0.662 to 0.838 . Cronbach's $\alpha$ after deletion ranged from 0.979 to 0.0980 , indicating high internal consistency among the topics; therefore, the topic selection was reasonable. The questionnaire data exhibited high reliability and could therefore be used to examine the results.

To explore the differences in evaluation of the film between age groups, 27 assessment elements were taken as dependent variables with age as the independent variable. Analysis of variance was performed, and the results are shown in Table 1. Age presented significant differences in the aforementioned evaluation elements. Comparison of the averages is shown as follows.

Presentation of image features C7-3: $4(5.00)>5(4.78)>1(4.05)>2(4.02)>3(4.00)$.

Presentation of life experience C8-2: $4(4.80)>5(4.33)>3(3.86)>1(3.69)>2(3.68)$.

Interpretation of role identity C8-1: $4(5.00)>5(4.22)>3(4.14)>1(3.83)>2(3.77)$.

Deep-rooted resonance C9-3: $4(4.80)>5(4.67)>3(4.00)>1(3.92)>2(3.91)$.

Empathy interaction B6-1: $4(4.60)>5(4.44)>3(4.28)>1(3.80)>2(3.75)$.

Among these significant features, the four characteristics, namely life experience, role identification, empathy, and depth of heart of the semantic layer, were consistent. The results with significant differences were for people aged 40-49 years, over 50 years, 30-39 years, younger than 19 years old, and 20-29 years; the image features of the technical layer were most significant, in order from least to most significant, for those aged $40-49$ years, older than 50 years, younger than 19 years, 20-29 years, and 30-39 years.

The cognitive difference of participants in different age groups after viewing a film is consistent with the results of other studies [41].

Table 1. Cognitive differences in evaluation of elements among age groups.

\begin{tabular}{|c|c|c|c|c|c|c|c|c|c|c|c|c|c|}
\hline $\mathbf{Q}$ & & SS & DF & MS & $\mathbf{F}$ & Scheffe & $\mathbf{Q}$ & & SS & DF & MS & $\mathbf{F}$ & Scheffe \\
\hline C8-1 & $\begin{array}{c}\text { BG } \\
\text { WG } \\
\text { Total }\end{array}$ & $\begin{array}{c}8.558 \\
100.835 \\
109.393\end{array}$ & $\begin{array}{c}4 \\
135 \\
139 \\
\end{array}$ & $\begin{array}{l}2.139 \\
0.747\end{array}$ & $2.864^{*}$ & & A3-1 & $\begin{array}{c}\text { BG } \\
\text { WG } \\
\text { Total }\end{array}$ & $\begin{array}{c}6.839 \\
92.733 \\
99.571\end{array}$ & $\begin{array}{c}4 \\
135 \\
139 \\
\end{array}$ & $\begin{array}{l}1.710 \\
0.687\end{array}$ & $2.489 *$ & \\
\hline
\end{tabular}




\subsection{Cognitive Differences between Professional Backgrounds}

To further explore the differences in the evaluation of the film's elements, professional background was used as an independent variable and 27 assessment elements were dependent variables. Analysis of variance was performed and the results are shown in Table 2. Professional backgrounds showed significant differences in the evaluation elements. The main difference of C7-1 emotion expression in the technical layer was that music-related majors exhibited a greater value than did design-related majors. The average numbers for the significant differences in the evaluation of the semantic layer A2-1 depth of emotional appeal were $2(4.83)>3(4.23)>1(4.13)>5(3.91)>$ 4 (3.88), corresponding to music-related professionals, other professionals, film and animation-related professionals, art-related professionals, and design-related professionals, respectively. The average numbers for the significant differences in the evaluation of B5-1 mood transfer were $2(4.50)>$ 5 (4.36) $>1(4.13)>3(4.09)>4$ (3.73), corresponding to music-related professionals, other professionals, film and animation-related professionals, art-related professionals, and design-related professionals, respectively. The average numbers for the significant differences in the evaluation of B6-1 empathy interaction were $2(4.50)>5(4.18)>3(4.11)>1(3.83)>4(3.66)(4.46)>3(4.11)>1(4.04)>4(3.78)$, and those for C9-3 deep-rooted resonance were $2(4.68)>5(4.46)>3(4.11)>1(4.04)>4(3.78)$, corresponding to music-related professionals, other professionals, art-related professionals, film and animation-related professionals, and design-related professionals, respectively. The total ratings of music preferences were significantly greater than those of film and animation. Music relatedness was significantly greater than that of design, showing that music-related professionals were significantly different from those of the other backgrounds.

Table 2. Cognitive differences in evaluation elements among professional backgrounds.

\begin{tabular}{|c|c|c|c|c|c|c|c|c|c|c|c|c|c|}
\hline $\mathbf{Q}$ & & SS & DF & MS & $F$ & Scheffe & $\mathbf{Q}$ & & SS & DF & MS & $F$ & Scheffe \\
\hline \multirow{3}{*}{ C7-1 } & BG & 6.988 & 4 & \multirow{3}{*}{$\begin{array}{l}1.747 \\
0.578\end{array}$} & \multirow{3}{*}{3.024 * } & \multirow{3}{*}{$2>4$} & \multirow{3}{*}{ A2-1 } & BG & 7.111 & 4 & \multirow{3}{*}{$\begin{array}{l}1.778 \\
0.619\end{array}$} & \multirow{3}{*}{$2.873 *$} & \\
\hline & WG & 77.983 & 135 & & & & & WG & 83.539 & 135 & & & \\
\hline & Total & 84.971 & 139 & & & & & Total & 90.650 & 139 & & & \\
\hline \multirow{3}{*}{ B5-1 } & BG & 7.988 & 4 & \multirow{3}{*}{$\begin{array}{l}1.997 \\
0.770\end{array}$} & \multirow{3}{*}{$2.595 *$} & & \multirow{3}{*}{ B6-1 } & BG & 8.486 & 4 & \multirow{3}{*}{$\begin{array}{l}2.121 \\
0.729\end{array}$} & \multirow{3}{*}{$2.909 *$} & \\
\hline & WG & 103.898 & 135 & & & & & WG & 98.450 & 135 & & & \\
\hline & Total & 111.886 & 139 & & & & & Total & 106.936 & 139 & & & \\
\hline \multirow{3}{*}{ C9-3 } & BG & 8.501 & 4 & \multirow{3}{*}{$\begin{array}{l}2.125 \\
0.752\end{array}$} & \multirow{3}{*}{$2.827 *$} & & \multirow{3}{*}{ B3 } & BG & 10.709 & 4 & \multirow{3}{*}{$\begin{array}{l}2.677 \\
0.648\end{array}$} & \multirow{3}{*}{$4.132 * *$} & \multirow{3}{*}{$\begin{array}{l}2>1 \\
2>4\end{array}$} \\
\hline & WG & 101.499 & 135 & & & & & WG & 87.463 & 135 & & & \\
\hline & Total & 110.000 & 139 & & & & & Total & 98.171 & 139 & & & \\
\hline
\end{tabular}

${ }^{*} p<0.05,{ }^{* *} p<0.01$; SS: Sum of Squares; MS: Mean Square; BG: Between Groups; WG: Within Groups

\subsection{Effects of Assessment Factors on Overall Assessment}

To further explore the effects of various elements of film and television on creativity and culture, the recognition of each element was an independent variable; high-quality creative intensity, cultural depth, and degree of preference for the work were dependent variables. Multiple regression was conducted and the results are shown in Table 3. The overall forecasting variable and the dependent variable coefficient $R$ of quality of creative intensity was 0.860 . The explanatory power of the quality of creative intensity of the 27 predictors was $73.9 \%$, and the $F$ value was 11.215 , reaching a significant correlation of 0.001 . The construction of the film "Like", spatial environment, use of performing skills, immersive relationship, empathetic interaction, strength of the mind conjunction, and quality of creative intensity were significantly and positively correlated, whereas the depth of emotional affection, elaboration of story content, theme cognition expression, and quality of creative intensity presented a significant negative correlation. Culturally specific personal in-depth overall prediction and dependent variable coefficient $R$ was 0.851 . The explanatory value of 27 predictors on cultural characteristics was $72.4 \%$. The $F$ value was 10.405 , achieving a 0.001 significant correlation. The use of film language, use of acting skills, development of story content, immersive relationship, and depth of cultural traits were significantly and positively related. The expression of image features, skills of 
emotional expression, interpretation of role identity, and depth of cultural traits were significantly negatively related; no significant difference was presented in taste preference.

Table 3. Cognitive differences in overall evaluation between evaluation elements.

\begin{tabular}{|c|c|c|c|c|}
\hline Antecedent & Independent Variable & B & $\beta$ & $t$ \\
\hline \multirow{8}{*}{ level of creativity } & construction of spatial environment & 0.182 & 0.192 & $2.047 *$ \\
\hline & presentation skills & 0.213 & 0.222 & $2.632 * *$ \\
\hline & the degree of the emotional appeal & -0.192 & -0.209 & -2.126 * \\
\hline & the development of the story & -0.254 & -0.267 & -2.493 * \\
\hline & theme communication & -0.179 & -0.185 & $-2.057 *$ \\
\hline & immersive relationship & 0.170 & 0.195 & $2.155 *$ \\
\hline & empathetic interaction & 0.218 & 0.258 & $2.353 *$ \\
\hline & deep-rooted sympathy & 0.259 & 0.310 & $2.599 *$ \\
\hline \multicolumn{5}{|c|}{$\mathrm{R}=0.860 ; \mathrm{Rsq}=0.739 ; \mathrm{F}=11.215^{* * *}$} \\
\hline \multirow{7}{*}{ depth of culture } & use of film language & 0.265 & 0.256 & $2.955^{* *}$ \\
\hline & image feature presentation & -0.231 & -0.217 & -2.196 * \\
\hline & presentation skills & 0.355 & 0.344 & $3.966^{* * *}$ \\
\hline & skills of emotional expression & -0.190 & -0.185 & -2.068 * \\
\hline & the development of the story & 0.335 & 0.326 & $2.967 * *$ \\
\hline & interpretation of role identity & -0.220 & -0.243 & -2.065 * \\
\hline & immersive relationship & 0.266 & 0.282 & $3.032 * *$ \\
\hline \multicolumn{5}{|c|}{$\mathrm{R}=0.851 ; \mathrm{Rsq}=0.724 ; \mathrm{F}=10.405^{* * *}$} \\
\hline
\end{tabular}

\section{Conclusions}

Environmental protection has become a topic of concern worldwide. This study provided the following insights:

1. People have a high degree of awareness of the themes in sand animation films, and cognitive resonance with topics can be aroused within them. They have a common understanding of environmental issues and think that environmental awareness, philosophy, action, and education should be strengthened to safeguard the living environment.

2. The participants were impressed by the images directly related to hunting and mangling in the animation. In particular, emotions were elicited by content related to human nature, and the new angle of film presentation was acceptable to the audience. Borrowing religious forms to express environmental issues was preferred by some and seen as controversial to others. However, it is believed that borrowing this perspective is more conducive to conveying the concept of environmental protection.

3. The use of fictional images to demonstrate environmental issues received unanimous praise from the participants. Through artistic, creative, subtle, and esthetic appearance, more praise can be gained. People prefer imaginative expression in the imagination space compared with photos and paintings. As long as the image form is highly compatible with the topic and interactive, the specific presentation form, realistic or freehand, is irrelevant. This is different from Edmund Burke's view that people devote more attention to real attributes. The result does not present unique tendencies of reality, but tendencies common to both realistic and freehand writing.

4. There were differences in perceptions of the film among different ages of viewers. The cognitive differences of life experience, interpretation of role identification, empathetic interaction, and sympathy were consistent. Significant differences were found, in sequence of scores from high to low, for participants 40-49 years old, 50 years old, 19 years old, 20-29 years old, and 30-39 years old. Older people had different cognitions of these items from younger audiences; there were also significant differences in the evaluations of the technical presentation of image features. The difference in results had similar properties to those four items. 
5. There were significant differences in the recognition of images among audiences of different professional backgrounds, which were embodied in the following aspects: skills of emotional expression, depth of emotional appeal, mood transfer, empathetic interaction, and deep-rooted resonance. Generally, there were significant cognitive differences between music-related professionals and other professionals.

6. The construction of a spatial environment, use of performing skills, immersive relationship, empathetic interaction, intensity of mind conjunction, and quality of creative intensity were positively correlated. The audience members with higher scores more effectively perceived the creative intensity of the image, whereas the depth of emotional appeal, development of the content of the story, and theme of the cognitive communication were significantly negatively correlated. The use of film language, use of presentation techniques, development of the content of the story, immersive relationship, and depth of cultural traits were significantly positively correlated. The expression of image features, skills of emotional expression, and interpretation of role identity were significantly negatively correlated.

7. The promotion and dissemination of environmental awareness through sand animation is effective and arouses people's concern for environmental protection. Concurrently, the depth of creativity and theme of communication exert a greater effect on communication. In the digital age, video media are ubiquitous. How to attract attention using audience-specific elements and evoke empathy is a topic worth exploring.

The current environmental situation is very serious. Government departments, companies, and individuals should further raise awareness of environmental protection, practice their own efforts, and work together to make a useful contribution to environmental protection. Effective communication of the theme of environmental protection through animation involves many elements. The composition of film and television art is complex. This is only a preliminary study, and the influence of elements such as music, script, and lens on theme implications requires further exploration.

Author Contributions: Writing: J.W.; providing the case and idea: Y.G. and R.L.; providing revision advice: S.-B.T. and'J.W.

Conflicts of Interest: The authors declare no conflict of interest.

\section{References}

1. Liu, T. Environmental Communication: Discourse, Rhetoric, 1st ed.; Peking University Press: Beijing, China, 2011; p. 1.

2. Clive, P.; Wang, Y. A Green History of the World: The Environment and Collapse of Great Civilization, 1st ed.; Shanghai People's Publishing House: Shanghai, China, 2002; p. 7.

3. Wang, R.H. Environmental Film: Communicating Green Development, a New Concept. J. Environ. Educ. 2016, 4, 51-53.

4. Qi, Y.L.; Qiao, J.M. Study on the Ecological Image of Environmental Films. China Youth Soc. Sci. 2017, 36, $128-134$.

5. Chen, G.L. Type Analysis of Environmental Films and Ecological Appeals. Ecol. Bull. 2014, 49, 41-45.

6. Tong, T. On the Aesthetic Value of Animation Art. Modern Communication. J. Commun. Univ. China 2005, 5, 65-67.

7. $\mathrm{Xu}, \mathrm{R}$. On the Function of Micro Animation and Its Cultural Responsibility. China Press 2013, 10, 76-77.

8. Scott, M.D. Toward an Eco-Cinema. Interdiscip. Stud. Lit. Environ. 2004, 11, 107-132.

9. Kenyon, H. Animating under the Camera. In Animat. Mag. 1998, 3.

10. Kazi, R.H.; Chua, K.C.; Zhao, S.; Davis, R.; Low, K.L. SandCanvas: A multi-touch art medium inspired by sand animation. In Proceedings of the SIGCHI Conference on Human Factors in Computing Systems, Vancouver, BC, Canada, 1-7 May 2011; pp. 1283-1292.

11. Wu, J. Development and Characterization of Experimental Sand Animation, World Chin. Paint. Calligr. 2017, 3, 82-84.

12. Shih, C.J. An Evolutionary Synthesis of Art and Technology; Department of Cultural Affairs: Taipei City, Taiwan, 2010; Volume 3, p. 43.

13. William, R. Literature and Ecology: An Experiment in Ecocriticism. Iowa Rev. 1978, 9, 71-86. 
14. Chen, Y. Real Dimensions of Ecological Film Criticism in China. Modern Lit. Circle 2015, 2, 103-107.

15. Shao, D. Ecological Film Aesthetics Research from the Perspective of "Rendering and Reconstruction-All Ecology". Sichuan Opera 2015, 3, 89-91.

16. Alexander, V.D. Sociology of the Arts Exploring Fine and Popular Forms, 1st ed.; Blackwell Publishing Ltd.: Oxford, UK, 2003; pp. 193-210.

17. Wendy, G. Rencent Moves in the Sociology of Literature. Annu. Rev. Sociol. 1993, 19, 455-457.

18. John, F. Reading the Popular, 1st ed.; Routledge: London, UK, 1990; pp. 130-156.

19. Stuart, H. Culture, Media, Languages, 1st ed.; Hutchinson: London, UK, 1980; pp. 30-80.

20. Burke, E. A Philosophical Enquiry into the Origin of Our Ideas of the Sublime and Beautiful, 1st ed.; Daxiang Press: Henan, China, 2010; p. 43.

21. McLuhan, M. Understanding Media-The Extensions of Man, 1st ed.; Commercial Press: Shanghai, China, 2000; pp. 33-50.

22. Lasswell, H.D. The Structure and Function of Communication in Society, 1st ed.; Harper \& Bros: York, PA, USA, 1948.

23. Harper, D. Meaning and Work: A Study in Photo Elicitation. Curr. Sociol. 1986, 34, 24-46. [CrossRef]

24. Wang, C.C.; Burris, M.A. Photovoice: Concept, Methodology, and Use for Participatory Needs Assessment. Health Educ. Behav. 1997, 24, 369-387. [CrossRef] [PubMed]

25. Collier, J.; Collier, M. Visual Anthropology: Photography as a Research Method; University of New Mexico Press: Albuquerque, NM, USA, 1986.

26. Goldman, A. Evaluating art. In The Blackwell Guide to Aesthetics; Wiley-Blackwell: Oxford, UK, 2004; pp. 93-108.

27. Trivedi, S. Artist-audience communication. Tolstoy Reclaimed 2004, 38, 38-52.

28. Lin, R.; Lin, P.H.; Shiao, W.S.; Lin, S.H. Cultural Aspect of Interaction Design beyond Human-Computer Interaction. In International Conference on Internationalization, Design and Global Development; Springer: Berlin/Heidelberg, Germany, 2009; pp. 49-58.

29. Chen, S.J.; Lin, C.L.; Lin, R. The Study of Match Degree Evaluation between Poetry and Paint. In Proceedings of the 5th Asian Conference on the Arts and Humanities (ACAH 2014), Osaka, Japan, 3-6 April 2014.

30. Chen, S.J.; Lin, C.L.; Lin, R. A Cognition Study of Turning Poetry into Abstract Painting. In Proceedings of the Fifth Asian Conference on Cultural Studies (ACCS 2015), Kobe, Japan, 28-31 May 2015.

31. Gao, Y.J.; Chen, L.Y.; Lee, S.; Lin, R.; Jin, Y. A Study of Communication in Turning "Poetry" into "Painting". In International Conference on Cross-Cultural Design; Springer: Cham, Switzerland, 2017; pp. 37-48.

32. Lin, R.; Qian, F.; Wu, J.; Fang, W.T.; Jin, Y. A Pilot Study of Communication Matrix for Evaluating Artworks. In International Conference on Cross-Cultural Design; Springer: Cham, Switzerland, 2017; pp. 356-368.

33. Craig, R.T. Communication theory as a field. Commun. Theory 1999, 9, 119-161. [CrossRef]

34. Fiske, J. Introduction to Communication Studies, 1st ed.; Routledge: London, UK, 2010.

35. Jakobson, R. Language in Literature, 1st ed.; The Belknap Press of Harvard U.P.: Cambridge, UK, 1987.

36. Hsu, C.H.; Chang, S.H.; Lin, R. A design strategy for turning local culture into global market products. Int. J. Affect. Eng. 2013, 12, 275-283. [CrossRef]

37. Lin, R. Transforming Taiwan Aboriginal Culture Features into Modern Product Design-A Case Study of Cross Cultural Product Design Model. Int. J. Design 2007, 1, 47-55.

38. Lin, R.; Kreifeldt, J.; Hung, P.H.; Chen, J.L. From Dechnology to Humart-A Case Study of Taiwan Design Development. In Cross-Cultural Design Methods, Practice and Impact; Springer International Publishing: Cham, Switzerland, 2015; pp. 263-273.

39. Norman, D.A. Emotional Design: Why We Love (or Hate) Everyday Things, 1st ed.; Basic Books: New York, NY, USA, 2005.

40. Chen, H.W. The Study on Features of Animal Characters in Animation Films. J. Perform. Vis. Arts Stud. 2014, 7, 29-42.

41. Jun, W.; Chang, T.C.; Yang, G.; Rugtai, L. A Case Study of Difference between Sand Animation and Micro Film. In Proceedings of the 7th International Conference on Kansei Engineering \& Emotion Research (KEER 2018), Kuching, Malaysia, 19-22 March 2018.

(C) 2018 by the authors. Licensee MDPI, Basel, Switzerland. This article is an open access article distributed under the terms and conditions of the Creative Commons Attribution (CC BY) license (http://creativecommons.org/licenses/by/4.0/). 